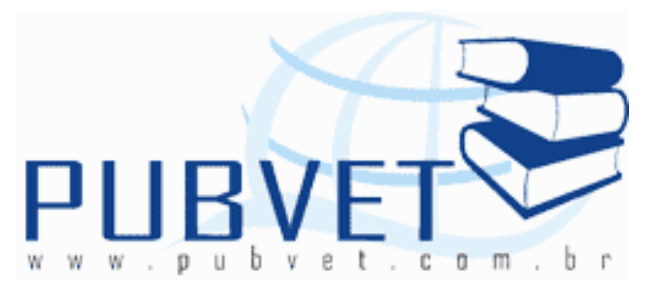

PUBVET, Publicações em Medicina Veterinária e Zootecnia.

\title{
Anatomia vascular de Phrynops geofroanus. Estudo da artéria aorta.
}

André Luiz Quagliatto Santos ${ }^{\mathbf{1}}$, Lorena Tannús Menezes², Janine Denadai ${ }^{2}$, Antonio Vicente Mundim ${ }^{1}$, Arthur Paulino Sanzo Kaminishi ${ }^{2}$, Tatiana Grillo Leonardo $^{2}$, Liliane Rangel Nascimento ${ }^{2}$

Laboratório de Ensino e Pesquisas em Animais Silvestres - LAPAS, FAMEV/UFU, e-mail: quagliatto@famev.ufu.br 1. Docentes. 2. Mestrandos.

\section{Resumo}

Estudou-se o comportamento da artéria aorta, focalizando sua origem, trajeto, e destino de suas ramificações. Utilizaram-se cinco exemplares de Phrynops geofroanus, de ambos os sexos, adultos, capturados no rio Uberabinha, município de Uberlândia-MG, com a licença de pesca científica- categoria D n163E DPB/IEF. As artérias carótidas comuns foram canuladas através das quais introduziu solução fisiológica a fim de desobstruir o sistema vascular, em seguida foi aplicado solução de Neoprene Látex "450" corada. Fixaram o material em solução de Formol a 10\% por um período de 96 horas, após este desprenderam a carrapaça de suas inserções, evidenciando as vísceras, e individualizou-se a artéria aorta. Foi observado que os arcos aórticos direito e esquerdo unem-se em um ponto após sua origem no coração. O arco aórtico esquerdo emite a artéria gástrica, que surge cranialmente ao ponto de união dos arcos aórticos. Emite a artéria celíaca, que fornece as artérias pancreaticoduodenais cranial e caudal e as artérias mesentéricas cranial. Após, 
o tronco formado pela união dos dois arcos aórticos, denomina-se artéria aorta e desta originam-se as artérias renais, epigástricas, ilíacas comuns e a artéria caudal, que é um fino segmento da porção final da artéria aorta. O arco aórtico direito não emite nenhum ramo antes da sua união com o arco aórtico esquerdo, portanto os principais ramos viscerais surgem do arco aórtico esquerdo ou diretamente da artéria aorta.

Palavras-chave: artérias, cágado-de-barbicha, artéria aorta, répteis.

\section{Vascular anatomy of the Phrynops geofroanus. Aorta artery study.}

\section{Abstract}

Study the behave of the artery aorta, to focus its origin, trajectory, and destiny of its ramifications. Was used five exemplars of Phrynops geofroanus, males and females, adults, from the Uberabinha river, Uberlândia-MG with the scientific fishing license category Dn- 163E DPB/IEF. Was tube the common carotid arteries through which we introduce a physiological solution to remove obstruction from the vascular system, than we inject solution of Neoprene Latex " 450 " colored. Was fixating the material in solution of Formaldehyde $10 \%$ for a period of 96 hours. After this, loose the skull from its insertions, showing the viscera, and individualizing the artery aorta. Were observed that the right and left aortic arcs unite in a point after the origin in the heart. The left aortic arch send the gastric artery, that arise cranial to the point of union of the aortic arcs. It still arises a celiac artery, that provides the cranial and caudal pancreaticduodenal arteries and the cranial and caudal mesenteric arteries. Right after, the trunk formed by the union of the two aortic arcs, is denominated the artery aorta and from it originates the renal arteries, epigastrics, common iliacs and the caudal artery, that is a thin segment of the final portion of the aorta. The aortic right arc does not emit any branch before its union with the left aortic arc, so the main visceral branches comes from the left aortic arc or directly from the aorta artery.

Keywords: arteries, tortoise, artery aorta, reptiles. 
SANTOS, A.L.Q. et al. Anatomia vascular de Phrynops geofroanus. Estudo da artéria aorta. PUBVET, Londrina, V. 5, N. 14, Ed. 161, Art. 1091, 2011.

\section{INTRODUÇÃO}

Os répteis provocam todos os tipos de reações nas pessoas, uma vez que são grupos de animais pouco compreendidos e escassamente conhecidos.

Nos dias de hoje podemos considerar que os répteis são pouco numerosos, pois se comparando ao período cretáceo, por exemplo, denominado de"A idade dos répteis", de 135 a 70.000.000 anos atrás, eram os animais dominantes na Terra (BURTON e BURTON, 1984; CARR, 1981; FUENTE, 1982; ORR, 1986; PRITCHARD, 1993).

Após esta era, a maioria sucumbiu, mas os que conseguiram sobreviver evoluíram em dura competição com aves e mamíferos, modificando suas estruturas e alterando sua fisiologia até conseguir uma adaptação em quase todos os biótipos terrestres e aquáticos do mundo contemporâneo (FUENTE, 1982). Porém é uma classe pouco estudada se comparada a peixes, aves e mamíferos.

A Phrynops geofroanus, é um quelônio da fauna brasileira e um dos maiores de água doce da América do Sul, o qual pertence ao filo Chordata, ao subfilo Vertebrata, a superclasse Tetrapoda, a classe Reptila e a ordem Chelonia (AMABIS e MARTHO, 1990; ASHLEY, 1969; BENZZONI, 1972; GRASSÉ, 1978; HICKMAN e MORANDINI, 1968; RENNER e CUNHA, 1969; ROMER, 1973; ROMER e PARSONS, 1985; ORR, 1986; POUGH et al., 1993; STORER et al., 1995; ZISWILLER, 1978).

Como características gerais, a Phrynops geofroanus, apresenta um corpo inteiramente coberto por um casco, também denominado carapaça superior ou casco e o plastrão na porção inferior, que são fortes de onde somente a cabeça, os membros e a cauda emergem. A carapaça e o plastrão são formados por placas ósseas poligonais, soldadas.

De acordo com Breland (1953), o arco sistêmico origina as artérias gástricas, celíaca e mesentérica superior, onde a artéria gástrica supre o estômago, a celíaca o duodeno, pâncreas, parte posterior do estômago e estruturas associadas, e a mesentérica superior o intestino, e ainda relata que 
SANTOS, A.L.Q. et al. Anatomia vascular de Phrynops geofroanus. Estudo da artéria aorta. PUBVET, Londrina, V. 5, N. 14, Ed. 161, Art. 1091, 2011.

a aorta dorsal origina a artéria ilíaca interna que irriga o intestino grosso. Harrison (1964) relata que a aorta dorsal dá origem à artéria celíaca a qual origina o ramo gástrico anterior, o ramo pancreaticoduodenal e o ramo hepático para seus respectivos órgãos; e a artéria mesentérica anterior para o fígado e intestino delgado. Baroudi (1965) afirma que as duas aortas depois de formarem a respectiva crossa, unem-se formando a aorta torácica única, que desce pelo tronco emitindo ramos em todo o seu trajeto e irrigando as estruturas do organismo. Nielsen (1986); Storer et al. (1995) relatam que os dois arcos aórticos unem-se numa aorta dorsal que distribui sangue para os órgãos da cavidade do corpo, para os membros posteriores e para a cauda. Entretanto Neal e Rand (1954); Hyman (1957) comentam que o arco aórtico esquerdo origina as artérias celíaca e mesentérica anterior, as quais carreiam sangue até o estômago e intestino, ainda cita que os arcos aórticos direito e esquerdo originan-se do ventrículo direito e esquerdo respectivamente, unindo-se em um tronco comum próximo da parte caudal do estômago. Faria e Mariana (2001) cita que a partir da artéria celíaca tem origem as artérias mesentérica inferior e superior.

Levando-se em consideração a importância do conhecimento do sistema circulatório em especial o comportamento da artéria aorta destes animais, que funciona como um meio de transporte do sangue rico em nutrientes (sais minerais, carboidratos, aminoácidos, vitaminas, proteínas, etc.), excretas, hormônios e componentes do sistema imunológico, é que o Laboratório de Pesquisa em Animais Silvestres da Faculdade de Medicina Veterinária da Universidade Federal de Uberlândia visa, com a presente pesquisa, descrever a origem e distribuição de seus principais ramos na cavidade celomática, bem como enriquecer a literatura sobre a Anatomia de Répteis, visto que, a literatura sobre o assunto é escassa e imprecisa limitando-se a aspectos biológicos. 
SANTOS, A.L.Q. et al. Anatomia vascular de Phrynops geofroanus. Estudo da artéria aorta.

PUBVET, Londrina, V. 5, N. 14, Ed. 161, Art. 1091, 2011.

\section{MATERIAL E MÉTODO}

Utilizamos cinco exemplares de Phrynops geofroanus, de ambos os sexos e adultas. Estes quelônios foram capturados no rio Uberabinha, no município de Uberlândia, MG, com a licença de pesca científica- categoria D n- 163E DPB/IEF Identificamos estes animais e encaminhamos ao Laboratório de Pesquisa em Organismos Aquáticos do Laboratório de Pesquisa em Animais Silvestres da Faculdade de Medicina Veterinária da Universidade Federal de Uberlândia, onde dissecamos e canulamos as artérias carótidas comuns com cânulas de polietileno de $2 \mathrm{~mm}$ de diâmetro por $50 \mathrm{~mm}$ de comprimento, através das quais introduzimos solução fisiológica a fim de desobstruir (lavar) o sistema vascular, em seguida aplicamos solução de Neoprene Látex "450" (Du Pont do Brasil S.A- Indústrias Químicas) corada com pigmento específico (Globo S/A Tintas e Pigmentos).

Fixamos o material assim preparado em solução aquosa de formol a $10 \%$ por um período mínimo de 96 horas. Após completado o tempo de fixação retiramos o plastrão do animal, desprendendo-o da carapaça e das suas inserções na região cervical, nos membros e na cauda. Feito isto, as vísceras ficaram expostas e presas apenas na carapaça, onde observamos a artéria aorta bem como seus principais ramos. A partir deste momento procedemos a dissecação com pinças, bisturi e tesouras cirúrgicas e utilizamos lupa Wild (10X) para melhor visualização da artéria aorta. Focalizamos da sua origem até sua terminação registrando-se suas relações, e o comportamento de cada um dos seus ramos. Algumas fotografias foram elaboradas para documentação dos resultados (Figuras 1 e 2 ).

\section{RESULTADOS}

Os resultados apresentados abaixo se basearam em observações macroscópicas realizadas pela vista dorsal dos animais. 
SANTOS, A.L.Q. et al. Anatomia vascular de Phrynops geofroanus. Estudo da artéria aorta. PUBVET, Londrina, V. 5, N. 14, Ed. 161, Art. 1091, 2011.

Analisando as peças dissecadas, pudemos verificar que os arcos aórticos direito e esquerdo unem-se em um ponto, logo após deixarem o ventrículo, obedecendo ao trajeto, inicialmente dorso-cranial e posteriormente ventrocaudal nas imediações da porção cardíaca do estômago. Próximo ao ponto de união dos dois arcos aórticos (na porção pilórica) e cranialmente a este, o arco aórtico esquerdo emite ramos para a irrigação do estômago e para algumas vísceras da cavidade celomática, enquanto que o arco aórtico direito não emite nenhum ramo antes da sua união com o arco aórtico esquerdo. Após a união dos arcos aórticos ocorre a formação da artéria aorta, de onde se originam ramos para a região médio-caudal do corpo do animal.

Para melhor descrevermos os trajetos destes vasos, optamos por fazê-lo separadamente desde sua origem do coração.

\section{ARTÉRIA GÁSTRICA}

A artéria gástrica surge da artéria pancreaticoduodenal cranial, que emerge do arco aórtico esquerdo, emite ramos para o estômago e baço.

\section{ARTÉRIA CELÍACA}

A artéria celíaca originou-se a partir do arco aórtico esquerdo em sua porção terminal, tendo aquela um menor calibre, seguindo na direção caudolateral esquerda, passando ventralmente a artéria aorta, iniciando a emissão de vários ramos a partir da região mediana, dos quais identificamos:

\section{ARTÉRIA PANCREATICODUODENAL CRANIAL:}

É o primeiro ramo a emergir da face lateral esquerda da artéria celíaca, seguindo em direção crânio lateral esquerda sendo envolvida pelo baço e envia a este ramos esplênicos também para o estômago.

\section{ARTÉRIA PANCREATICODUODENAL CAUDAL:}


SANTOS, A.L.Q. et al. Anatomia vascular de Phrynops geofroanus. Estudo da artéria aorta. PUBVET, Londrina, V. 5, N. 14, Ed. 161, Art. 1091, 2011.

Parte da face lateral esquerda da artéria celíaca, em direção caudo-lateral direito, enviando um ramo para o pâncreas e duodeno e outro para o fígado.

\section{ARTÉRIA MESENTÉRICA CRANIAL:}

Emerge em direção caudo-lateral direita, sendo responsável pela nutrição do mesentério, intestino delgado e parte do intestino grosso.

\section{ARTÉRIA MESENTÉRICA CAUDAL:}

Apresentou-se como continuação da artéria celíaca, sendo responsável pelo abastecimento sanguíneo de parte do intestino grosso e mesentério.

\section{ARTÉRIA AORTA}

Denomina-se de artéria aorta, a artéria que se inicia à partir da junção dos arcos aórticos esquerdo e direito. Tem um importante papel no suprimento de sangue da região médio-caudal do corpo do animal, uma vez que dela partem artérias para as vísceras, membros pelvinos, gordura lateral, músculos longitudinais, casco e cauda.

\section{ARTÉRIAS RENAIS}

Partindo da face ventral da artéria aorta, em direção aos rins, direito e esquerdo, observaram-se artérias renais direita e esquerda em número de 04 (duas) para cada rim.

Para ambos os rins encontraram-se a partir das artérias ilíacas comuns esquerda e direita, um ramo renal para cada órgão.

ARTÉRIAS GENITAIS:

Chamadas de ovarianas nas fêmeas ou testiculares nos machos, as artérias genitais surgiram à partir das artérias renais, próximas ao hilo renal, sendo uma para cada gônada. 
SANTOS, A.L.Q. et al. Anatomia vascular de Phrynops geofroanus. Estudo da artéria aorta. PUBVET, Londrina, V. 5, N. 14, Ed. 161, Art. 1091, 2011.

\section{ARTÉRIAS EPIGÁSTRICAS}

Acompanhando-se o trajeto da artéria aorta, logo após as artérias renais, encontramos a origem das epigástricas esquerda e direita. Estas seguiam em direção lateral esquerda e direita respectivamente e se bifurcavam em artérias marginocostal cranial e marginocostal caudal para seguir em direção crâniolateral e caudo lateral respectivamente, irrigando as margens do casco e músculos pélvicos.

\section{ARTÉRIAS ILÍACAS COMUNS}

A artéria aorta, em sua porção terminal emite as artérias ilíacas comuns esquerda e direita.

As artérias ilíacas comuns esquerda e direita constituem troncos comuns e emitem à partir de suas faces laterais direita e esquerda um ramo renal e um ramo lateral, antes de formar as artérias ilíacas internas e externas.

As artérias ilíacas interna e externa se destinam para parte do sistema genital e bexiga urinária.

As artérias ilíacas externas direita e esquerda apresentam como continuação das artérias ilíacas comum direita e esquerda e emitem 02 (dois) ramos, isto é as artérias femoral e isquiádica.

\section{ARTÉRIA CAUDAL}

A artéria caudal é um fino segmento mediano da porção final da artéria aorta, originando-se logo após a origem das epigástricas comuns. 
SANTOS, A.L.Q. et al. Anatomia vascular de Phrynops geofroanus. Estudo da artéria aorta.

PUBVET, Londrina, V. 5, N. 14, Ed. 161, Art. 1091, 2011.

As figuras 1 e 2 mostram a artéria aorta e seus principais ramos:

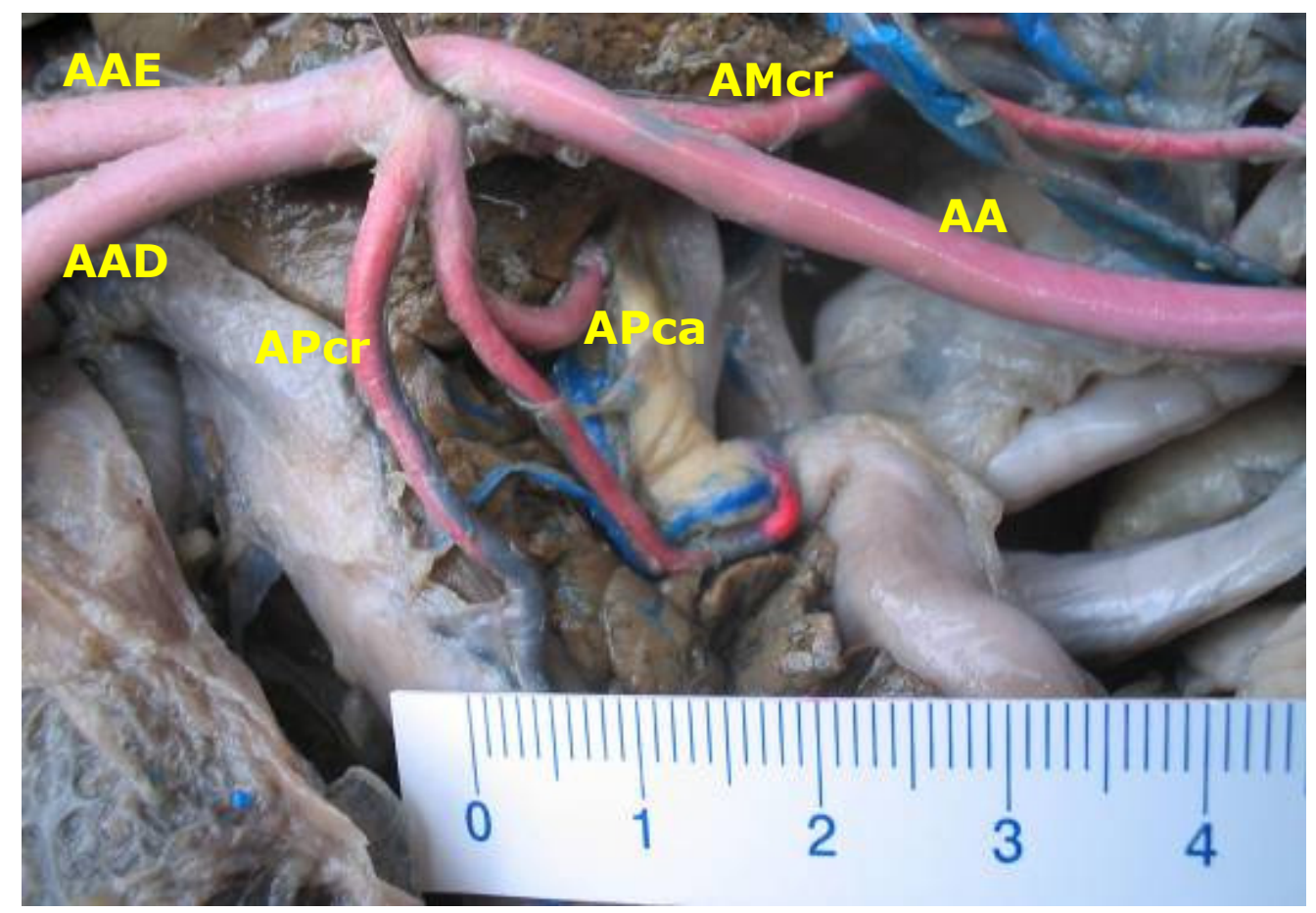

Figura 1: Fotografia de Phrynops geofroanus, (AAD) arco aórtico direito, (AAE) arco aortico esquerdo, (AA) artéria aorta, (AMcr) artéria mesentérica cranial, (APcr) artéria pancreaticoduodenal cranial, (APca) artéria pancreaticoduodenal caudal. 


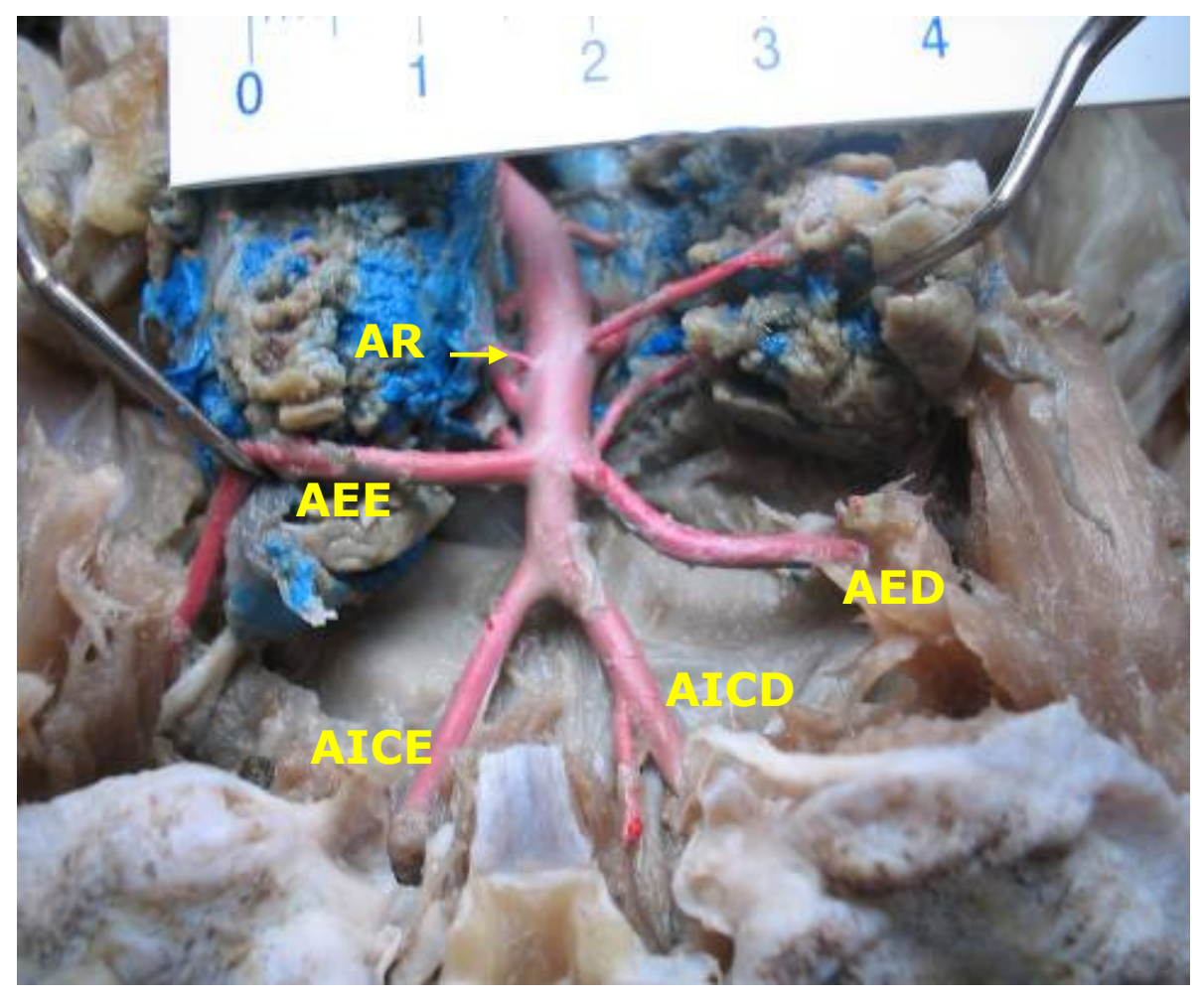

Figura 2: Fotografia de Phrynops geofroanus, (AR) artérias renais, (AED) artéria epigástrica direita, (AEE) artéria epigástrica esquerda, (AICD) artéria ilíaca comum direita, (AICE) artéria ilíaca comum esquerda.

\section{DISCUSSÃO}

Confrontando os resultados com as descrições de outros autores, concordamos com Neal e Rand (1954) e Oliveira et al. (2009) que ao descreverem sobre a origem e trajeto dos arcos aórticos direito e esquerdo afirmam que estes arcos após deixarem o coração possuem um trajeto dorsocranial e posteriormente ventro-caudal próximo a parte cranial do estômago, tendo um ponto de união entre os arcos aórticos direito e esquerdo, próximo a parte caudal do estômago.

Moraes e Santos (2004), que estudaram a Podocnemis expansa, também 
SANTOS, A.L.Q. et al. Anatomia vascular de Phrynops geofroanus. Estudo da artéria aorta. PUBVET, Londrina, V. 5, N. 14, Ed. 161, Art. 1091, 2011.

afirmam que a artéria mesentérica cranial é responsável pela nutrição do mesentério, intestino delgado e parte do intestino grosso. No entanto, os autores relatam que a artéria mesentérica caudal é uma continuação da artéria celíaca. Rodrigues, Miglino e Melo (2003) encontraram a artéria mesentérica caudal originando-se, na maioria dos casos, da artéria ilíaca interna do antímero direito. Faria e Melo (2007) em Trachemys scripta elegans a artéria mesentérica cranial executa o maior aporte da irrigação sanguinea do baço. No entanto, em $P$. geofroanus a artéria mesentérica caudal origina-se da artéria aorta.

Santos et al. (2003) também estudaram a anatomia vascular em Podocnemis expansa, mas o enfoque foi descrever as artérias coronárias, encontrando uma única artéria originada da face lateral direita do coração, na porção inicial do tronco braquiocefálico.

No que diz respeito ao arco aórtico esquerdo temos que Neal e Rand (1954), o presente trabalho relatam que o arco aórtico esquerdo origina a artéria celíaca, porém a mesentérica anterior também se origina do arco aórtico esquerdo, já Faria e Mariana (2001) informa que a mesentérica cranial apresenta-se como um segmento a partir da celíaca.

Santos et al. (2004) em Podocnemis expansa encontrou-se a artéria mesentérica cranial como um ramo do tronco celíaco-mesentérico e originando as artérias pancreaticoduodenal caudal que irrigam o duodeno; e a artéria mesentérica caudal origina-se da aorta ou da artéria ilíaca comum. Para Faria e Mariana (2001) em Geochelone carbonaria a artéria aorta esquerda ao invés de arco aórtico esquerdo e artérias mesentéricas inferior e superior não utilizando mesentéricas caudal e cranial.

Breland (1953) também afirma que a artéria celíaca origina-se do arco aórtico, porém este arco é denominado de arco sistêmico, não dando ênfase no arco aórtico esquerdo.

Rodrigues, Miglino e Melo (2003) estudaram a vascularização artéria do trato gastrointestinal da Trachemys scripta elegans e verificaram que a artéria pancreaticoduodenal caudal é originada da artéria hepática comum. Faria e 
SANTOS, A.L.Q. et al. Anatomia vascular de Phrynops geofroanus. Estudo da artéria aorta. PUBVET, Londrina, V. 5, N. 14, Ed. 161, Art. 1091, 2011.

Mariana (2001) encontraram a artéria pancreaticoduodenal caudal como sendo uma continuação da artéria celíaca, responsável pelo abastecimento sanguíneo do intestino grosso. Já em Phrynops geofroanus avaliou-se que a artéria pacreaticoduodenal caudal é originada da artéria mesentérica cranial.

Contrariando os resultados encontrados, Harrison (1964) descreve que é a partir da artéria aorta dorsal que se tem a artéria celíaca. O autor comenta também que á artéria celíaca origina o ramo gástrico anterior, o ramo pancreaticoduodenal e o ramo hepático para os respectivos órgãos, e a artéria mesentérica anterior para o fígado e intestino delgado. Já a pesquisa presente relata que é o arco aórtico esquerdo o responsável pelo surgimento da artéria pancreaticoduodenal cranial e esta por sua vez a gástrica, sendo as artérias pancreaticoduodenais cranial e caudal (irrigam baço, estômago e pâncreas e duodeno, respectivamente) originárias da artéria celíaca.

Hirano et al. (2009), estudando a Podocmenis unifilis observaram que as artérias renais se originam da face ventral da artéria aorta dorsal, e foram encontrados dois pares de artérias renais para cada rim.

Pereira et al. (2008) neste trabalho descrevem que a partir da união dos arcos aórticos direito e esquerdo tem-se a artéria aorta, entretanto, Baroudi (1965) relata a presença de duas aortas antes da união das mesmas, e após esta união tem-se a artéria torácica única que emite troncos e irriga as estruturas do organismo.

Storer et al. (1995) comentam a existência de dois arcos aórticos que se unem e forma a aorta dorsal, contrariando em parte, as nossas observações, pois esta, apontam para a presença da artéria aorta.

\section{CONCLUSÕES}

Com base nos dados do presente estudo conclui-se que:

A origem dos arcos aórticos obedece ao padrão reptiliano, onde o arco aórtico direito deixa o coração pelo lado esquerdo e o arco aórtico esquerdo pelo lado direito do ventrículo. 
SANTOS, A.L.Q. et al. Anatomia vascular de Phrynops geofroanus. Estudo da artéria aorta. PUBVET, Londrina, V. 5, N. 14, Ed. 161, Art. 1091, 2011.

O arco aórtico esquerdo origina a artéria celíaca, já o arco aórtico direito não emite nenhum ramo antes da união com o arco aórtico esquerdo.

A artéria aorta emite as artérias renais, epigástricas, ilíacas comuns e caudal.

\section{REFERÊNCIAS}

AMABIS, J. M.; MARTHO, G. R. Curso básico de biologia - seres vivos. São Paulo: Moderna, 1990. v.2. 388p.

ASHLEY, L. M. Laboratory anatomy of the turtle. Dubuque, Iowa: WM. C. Brown, 1969. 2634p.

BAROUDI, R. Elementos de zoologia de cyclostomata a mammalia. 4ed. São Paulo: Nobel, 1965. v.3.67p.

BENZZONI, P. Compêndio de zoologia. Belo Horizonte: Livraria Cultural Brasileira, 1972. $175 p$.

BURTON, M.; BURTON, R. Encyclopedia of reptiles, anphibians and other cold-blooded animals. San Sebastian: Tonsa, 1984. 121p.

BRELAND, O. P. Manual of comparative anatomy. 2ed. Nova York: McGraw-Hill Book, 1953. $124 p$.

CARR, A. Biblioteca da natureza- life- os répteis. Rio de Janeiro: Livraria José Olympio, 1981. 151p.

FARIA, T. N.; MARIANA, A. N. B., Origem e ramificações das artérias aortas esquerda e dorsal do jabuti (Geochelone carbonaria, Spix, 1824). Brazilian Journal of Veterinary Research and Animal Science. São Paulo, v.38, n. 4, p.155-159, Jan. 2001.

FARIA, M. D. ; MELO, A. P. F. Topografia, morfologia e irrigação do Baço em Trachemys scripta elegans (WIED, 1838). Brazilian Journal Veterinary Research Animal and Sciense, São Paulo, v. 44, n.3, p. 167-173, Out. 2007.

FUENTE, F. R. A fauna - vida e costume dos animais selvagens. Rio de Janeiro: Salvat, 1982. v.4. 57 p.

GRASSÉ, P. P. Zoologia - vertebrados reproducción, biologia, evolucion y sistemática agnatos, peces, anfibios y reptiles. Barcelona: Toray-masson, 1978. v. 3. 1-9p.

HICKMAN, C. P. Princípios de zoologia. (S.I): Ariel, s.d. 501-502 p.

HIRANO, L. Q. L.; SANTOS, A. L. Q.; PEREIRA, P. C.; SILVA, J. M. M.; KAMINISHI, Á. P. S.; FERREIRA, C. G. Anatomia vascular das artérias renais e gonadais de Podocnemis unifilis Schweigger, 1812 (Testudines, Pelomedusidae) Acta Scientiarum. Biological Sciences, Maringá, v.31, n.2, p.191-194, Fev. 2009. 
SANTOS, A.L.Q. et al. Anatomia vascular de Phrynops geofroanus. Estudo da artéria aorta. PUBVET, Londrina, V. 5, N. 14, Ed. 161, Art. 1091, 2011.

HYMAN, L. H. Comparative vertebrate anatomy. Chicago: The University of Chicago press, 1957. 347-348 p.

HARRISON, B. M. Manual of comparative anatomy a general laboratory guide. 2ed. St. Louis: C.V. Mosby, 1964. 156p.

MORAES, F. M.; SANTOS, A. L. Q. Anatomia vascular da Podocnemis expansa (TestudinataPelomedusidae). Comportamento da Artéria Aorta. Horizonte Científico. Uberlândia, n. 3, p. 1-13, Jan. 2004.

MORANDINI. C. Zoologia. 2 ed. São Paulo: Nobel S. A, 1968. v. 4. 1196. p.

NEAL, H.V.; RAND, H. W. Comparative anatomy. New York: Blakiston Company, 1954. 356p.

NIELSEN, K. S. Animal physiology: adaptation and environment. 3ed. New York: Cambridge University, 1986. 619p.

OLIVEIRA, S. C. R.; MACHADO JÚNIOR, A. A. N.; CARVALHO, R. C.; PEREIRA, L. A.; OLIVEIRA, A. S.; SOUSA, A. L. Distribuição arterial dos principais vasos da cavidade celomática em Kinosternon sorpioides scorpioides. Ciência Animal Brasileira, Goiânia, v.10, n.3, p.893898, Jul./Set. 2009

ORR, R. T. Biologia dos vertebrados. 5ed. São Paulo: Roca, 1986. 128-130 p.

PEREIRA, H. C. SILVA JUNIOR, L. M. MUNDIM, A. V.; SANTOS, A. L. Q. Anatomia vascular de Phrynops geoffroanus Schweigger, 1812 (Testudines-Chelidae)- origem e ramificações das artérias mesentérica cranial e mesentérica caudal In: VIII Encontro Interno e XII Seminário de Iniciação Científica, 0438, 2008, Uberlândia. Anais... Universidade Federal de Uberlândia, 2008, p. 1.

POUGH, F. H.; HEISER, J. B.; MCFARLAND, W. N. A vida dos vertebrados. São Paulo: Atheneu, 1993. 386- 409p.

PRITCHARD, P. C. H. Encyclopedia of turtles. New Jersey: T. F. H., 1973, 326p.

RENNER, M., CUNHA, A. X. Guia de trabalhos práticos de zoologia. 15ed. Coimbra: Atlântica, 1969. 341-415p.

RODRIGUES, R. F.; MIGLINO, M. A.; MELO, A. P. F. Vasculariazação arterial do trato gastrointestinal da Trachemys scripta elegans, Wied, 1838. Brasilian journal of veterinary research and animal science. São Paulo, n.40, p. 63-88, Out. 2003.

ROMER, A.S. Anatomia comparada (vertebrados). 4ed. México: Interamericana, 1973. 289-320p.

ROMER, A. S.; PARSONS, T. S. Anatomia comparada dos vertebrados. São Paulo: Atheneu, 1985. 381p.

SANTOS, A. L. Q.; ALVARENGA, G. J. R.; MORAES, F. M.; AVILA JUNIOR, R. H.; CARVALHO, S. F. M.; MAGALHÃES, L. M.; ANDRADE, M. B.; MARQUES, F. K.; DENADAI, J. Morfologia externa, topografia do coração e comportamento da artéria coronária de Podocnemis expansa (SCHWEIGGER, 1812). Bioscience Journal. Uberlândia, v. 19, n. 3, p. 103-108, Sept./Dec. 2003. 
SANTOS, A.L.Q. et al. Anatomia vascular de Phrynops geofroanus. Estudo da artéria aorta. PUBVET, Londrina, V. 5, N. 14, Ed. 161, Art. 1091, 2011.

SANTOS, A. L. Q.; MUNDIM, A. V.; MORAES, F. M.; ALVARENGA, G. J. R.; MARQUES, F. K.; BORGES, K. M.; ALVES JÚNIOR, J. R. F. Origem e ramificações das artérias mesentéricas cranial e caudal em tartaruga da amazônia Podocnemis expansa - Schweigger, 1812, (Testudinata-Pelomedusidae). Archives of Veterinary Science, Curitiba, v.9, n.2, p.49-53, Out. 2004.

STORER, T. I.; USINGER, R. L., STEBBINS, R. C.; NYBAKKEN, J. W. Zoologia geral. 6ed. São Paulo: Nacional, 1995. 642-667p.

ZISWILLER, V. Zoologia especial dos vertebrados. Barcelona: Omega, 1978. v.1. 1-3p. 